\title{
Percepción de factores que inciden en la participación y competencia de mujeres atletas en Para Powerlifting
}

Perception of factors that affect the participation and competition of female athletes in Para Powerlifting

\author{
Jenny Patricia Ortiz Cárdenas \\ Universidad El Bosque, Colombia \\ jocpcd@gmail.com. \\ (iD) https://orcid.org/0000-0003-2715-334 \\ Iván Ricardo Perdomo Vargas \\ Corporación Universitaria Minuto de Dios - UNIMINUTO, Colombia \\ iperdomovar@uniminuto.edu.co \\ (iD https://orcid.org/0000-0002-4753-3379
}

Giovanny Garzón Pastrana

Fundación Universitaria del Área Andina, Colombia

(DD https://orcid.org/0000-0003-3871-2372

Juan David Vargas Reyes

Fundación Universitaria del Área Andina, Colombia

https://orcid.org/0000-0002-7785-2557

\begin{abstract}
RESUMEN:
La mayoría de las personas con discapacidades físicas no participan en deportes regularmente y mucho menos en deporte de rendimiento. Por lo tanto, se necesita conocimiento sobre aquellos factores que inciden en la participación deportiva en los diferentes para deportes ofertados. Por esta razón, el presente estudio indagó en la percepción de dichos factores por parte de las mujeres para atletas que practican Para Powerlifting, teniendo como antecedente que de ese grupo poblacional ha existido menor crecimiento numérico a lo largo de los años. Para ello, se encuestaron 49 deportistas de 13 países participantes en el Bogotá 2018 World Para Powerlifting "Americas Open Championships". Se logró identificar que la práctica del Para Powerlifting es un motivador importante debido a su oportunidad de conseguir un desarrollo deportivo profesional, que barreras como la falta de escenarios deportivos y adecuación específica de acuerdo con las necesidades de las personas con discapacidad y del deporte son altamente percibidas por las para atletas que adicionalmente manifiestan que, a nivel gubernamental se han tomado medidas. Sin embargo, aún falta mayor trabajo para lograr la masificación del para deporte en la población femenina.
\end{abstract}

Palabras clave: Barreras, Facilitadores, Para Powerlifting, Para deporte, Para atletas, Mujer.

\begin{abstract}
:
Most people with physical disabilities do not participate in sports regularly, and much less in performance sports. Therefore, knowledge about those factors that affect sports participation in the different sports offered is required. For this reason, the present study investigated the perception of these factors by women para athletes who practice Para Powerlifting, taking as a precedent that there has been less numerical growth in this population group over the years. For this purpose, 49 para athletes from 13 countries participating in the Bogotá 2018 World Para Powerlifting "Americas Open Championships" were surveyed. It was possible to identify that the practice of Para Powerlifting is an important motivator due to its opportunity to achieve professional sports development. Barriers such as the lack of sports scenarios and specific adaptation according to the needs of people with disabilities and sports are highly perceived by the para athletes who additionally state that, at the governmental level, measures have been taken. However, more work is still needed to achieve the massification of para sport in the female population.
\end{abstract}

KeYwords: Barriers, Facilitators, Para Powerlifting, Para sport, Para athletes, Women. 


\section{INTRODUCCIÓN}

El deporte adaptado o para deporte, consiste en una modalidad deportiva que se ajusta a un grupo de personas con condiciones especiales de salud o discapacidades, con el fin de facilitar su práctica bien sea a través de una serie de adaptaciones o modificaciones o recurriendo a una estructura propia que permite su ejecución (Reina, 2010 en Moya, 2014). Si bien en sus inicios esta forma de práctica deportiva se concibió con fines terapéuticos, desde la década de los 60 se consolidó de manera competitiva a través de la celebración de los I Juegos Paralímpicos en Roma (Yagüe, 2008); al respecto, cabe señalar que este espacio cuenta con el mismo esquema, estructura y organización que los Juegos Olímpicos lo cual refleja un logro en términos de derechos e inclusión (Ruiz, 2012). .

En línea con lo anterior, las investigaciones realizadas en torno al impacto de la práctica deportiva en personas con algún tipo de discapacidad, sugiere que esta tiene un alcance positivo significativo en términos de calidad de vida y reintegración a la comunidad, al tiempo que se transforma en parte de la identidad del para atleta mediante la búsqueda de reconocimiento y reafirmación social (Jefferies, Gallagher y Dunne, 2012; Díaz, Miller, Kraus y Fredericson, 2019). Más aún, estos aspectos positivos se pueden reducir a seis grandes componentes que integran la calidad de la experiencia del para atleta y permiten una comprensión en profundidad de la misma: pertenencia, autonomía, desafío, maestría, participación y significado (Evans et al., 2018).

A pesar del reconocimiento que implica la práctica misma del deporte paralímpico en un nivel competitivo, existe un conjunto significativo de factores que dificulta la dinámica misma de esta modalidad. Entre las más frecuentes se encuentran el desconocimiento del deporte, la insuficiencia de personal capacitado y especializado, escenarios deportivos no adaptados, un nivel relativamente bajo de investigación en procesos de entrenamiento, limitaciones en el acceso a medios de transporte, entre otros (Ruiz, 2012). Aunado a lo anterior, aspectos personales como la gestión del tiempo es una barrera muy importante, pues implica equilibrar diferentes dimensiones de la vida como lo es el trabajo, compromisos personales y entrenamientos (McLoughlin, Weisman, Castaneda, Gwin y Graber, 2017). Adicionalmente, variables como la edad y el tipo de deporte practicado parecen influir en la percepción de dichas dificultades (Jaarsma, Dijkstra, Geertzen y Dekker, 2014).

Por otro lado, uno de los grandes facilitadores para la práctica del para deporte es el apoyo familiar (Camargo y Forero, 2016; Abellán y Januário, 2017); además, el alcanzar logros relacionados con el desempeño, junto con la posibilidad de contar con un entrenador que motive la participación y fomente la confianza en los para atletas, son algunos de los facilitadores individuales comúnmente referidos (McLoughlin, Weisman, Castaneda, Gwin y Graber, 2017; Camargo y Forero, 2016). En términos de diferencias de género, se ha encontrado que el apoyo de los amigos es un facilitador más importante para los hombres, mientras que el apoyo de los maestros lo es para las mujeres (Abellán y Januário, 2017).

Ahora bien, el deporte adaptado que se abordó en el presente trabajo corresponde al Para Powerlifting, el cual es el para deporte de fuerza por excelencia. Su inclusión en la historia del deporte paralímpico se remonta a 1964 en los Juegos Paralímpicos de Tokio. Inicialmente, este para deporte solo admitía atletas masculinos con lesiones de médula espinal; no obstante, en los años siguientes se incluyeron otros grupos con diversas discapacidades (World Para Powerlifting, s.f.). En términos generales, el Para Powerlifting se trata de una competencia realizada por para atletas con discapacidades físicas enmarcadas en ocho tipos propios de la discapacidad física; las personas tendrán entonces movilidad reducida con plena funcionalidad en el tren superior ya que el gesto técnico se desarrolla con ese segmento del cuerpo. Vale la pena mencionar que el Para Powerlifting es una de las disciplinas del programa de verano de los Juegos Paralímpicos que ha venido 
presentando el mayor crecimiento dentro de todos los para deportes pertenecientes al Comité Paralímpico Internacional, llegando a ser practicado en cerca de 100 países y con competiciones en 10 categorías diferentes (World Para Powerlifting, s.f.). Adicionalmente, variables como la edad y el tipo de deporte practicado parecen influir en la percepción de dichas dificultades (Jaarsma, Dijkstra, Geertzen y Dekker, 2014).

Específicamente, al intentar establecer el grado de participación de la mujer en este para deporte, no existen muchas investigaciones que hablen al respecto; en términos de la práctica deportiva en general, se evidencia la persistencia de actitudes estereotipadas hacia la mujer, las cuales incluyen imágenes de pasividad y delicadeza (Organización de Naciones Unidas, 2007; Wilde, 2007). Igualmente, otros aspectos de tipo estructural como la falta de apoyo institucional, así como barreras económicas y culturales asociadas con estructuras sociales patriarcales, entorpecen la participación de la mujer en el deporte (Farzaneh, Rahavi, Khalili, Khalili y Ranawat, 2021). Sin embargo, más allá de las especulaciones, es importante establecer las realidades que se observan desde las mujeres que actualmente hacen parte de este para deporte con el objeto de reconocer los factores que pueden influir en el grado de participación femenina y poder diseñar programas de impacto que permitan equilibrar dicha participación. Por tal razón, el presente trabajo busca una determinación primaria de la percepción de factores que inciden en la participación y práctica de este deporte en la región de las Américas, procurando así una oferta equitativa completamente incluyente.

\section{Metodología}

Diseño. El presente trabajo se enmarca desde el enfoque cuantitativo pues recurre a la recolección de datos y la medición estadística con el fin de dar cuenta de una aproximación a la realidad; adicionalmente, se desarrolla desde un diseño exploratorio pues busca examinar un tema poco estudiado como lo es el Para Powerlifting femenino (Hernández, Fernández y Baptista, 2014).

Participantes. La selección de la muestra se dio de manera no probabilística por conveniencia, teniendo en cuenta que para la selección de la misma se contó con la disponibilidad y disposición de las participantes a ser encuestadas (Hernández, Fernández y Baptista, 2014). De esta manera, de las 56 competidoras que participaron en el Bogotá 2018 World Para Powerlifting “Américas Open Championships”, se encuestaron a 49 mujeres Para Powerlifters de 13 países participantes, los abordados fueron Brasil (14), Chile (4), Colombia (5), Cuba (1), India (1), Japón (5), Kenia (3), Lituania (1), México (8), Perú (1), España (2), Turkmenistán (1) y Venezuela (3).

Instrumentos. Se utilizó como instrumento la "Encuesta de Barreras y Facilitadores para la práctica del Para Powerlifting para las Para Atletas Femeninas en la Región de las Américas”, documento dirigido a colectar la información acerca de la percepción de las barreras y facilitadores por parte de las para atletas de Para Powerlifting. La validez del instrumento se determinó mediante una evaluación de expertos, la cual refiere "al grado en que aparentemente un instrumento mide la variable en cuestión, de acuerdo con "voces calificadas”.” (Hernández, Fernández y Baptista, 2014 p. 204); en este sentido, la encuesta fue revisada por expertos de la Fundación Universitaria del Área Andina de la sede de Bogotá, Colombia, de las áreas de investigación, ciencias de la salud y entrenamiento deportivo. Posterior a la elaboración de la encuesta, fue revisada por los expertos, realizados los ajustes solicitados fue aprobada por estos y presentada junto con el cúmulo de documentos solicitados por el Comité Institucional de Ética en Investigación de la Fundación Universitaria del Área Andina, siendo esta aprobada por dicho comité dentro de la aprobación final y de respaldo de la investigación.

El instrumento se divide en dos partes, a las cuales precede el consentimiento informado conforme a la norma; la primera contiene seis interrogantes con múltiple respuesta, siendo un primer acercamiento hacia el reconocimiento de barreras y facilitadores que han tenido en la práctica del deporte; la segunda es una escala de Likert que puntúa entre 1. Muy poca importancia, 2. Poca importancia, 3. Importante, 4. Más que importante y 5. Muy importante, las barreras y facilitadores identificándose en dos grandes grupos: el 
Factor Personal (salud, psicológico, estado físico, familia, entorno y reconocimiento), y el Factor de Gestión Deportiva (promoción, infraestructura y capacitación). Los datos colectados fueron procesados por medio del paquete estadístico MATLAB 2014.

Procedimiento. La administración de la encuesta de recolección de datos se realizó dentro de los horarios de competencia del "Bogotá 2018 World Para Powerlifting Americas Open Championships" realizada del 6 al 9 de diciembre de 2018, con horarios de 10:00 a 20:00 horas del día. La divulgación de la investigación se llevó en primera instancia en la reunión técnico-informativa del evento a la cual por norma del deporte asisten dos delegados por país, los cuales recibieron una presentación de la investigación, los investigadores y los beneficios de la misma, se efectuó un acercamiento directo a los equipos por parte del equipo investigador, tras la aceptación de participación de las para atletas, les fue leído el consentimiento informado el cual fue firmado por cada participante, se realizó de manera personal con seguimiento de los investigadores.

Consideraciones éticas. Esta investigación garantiza la cooperación en investigación, la protección en el procesamiento de datos y se determinan la categoría de investigación sin riesgo. Se salvaguarda el Secreto profesional garantizando que los Investigadores se comprometen a no divulgar la identidad, ni los datos personales de las personas que voluntariamente participaron en el estudio. Igualmente, esta cuenta con la aprobación tanto del instrumento, como del consentimiento informado, procedimientos e investigación en general del Comité Institucional de Ética en Investigación de la Fundación Universitaria del Área Andina.

\section{RESULTADOS Y DISCUSIóN}

En la Tabla 1 se observa la frecuencia absoluta $(\mathrm{Fa})$ y la frecuencia relativa $(\mathrm{Fr})$ de las respuestas proporcionadas a los ítems relacionados con el constructo de acercamiento a la disciplina (ítems 1 al 4 ), el cual a su vez incluye un conjunto de factores como: (1) razones para la práctica del Para Powerlifting; (2) medios de acercamiento al deporte (3) preferencias para la práctica del Para Powerlifting y (4) beneficios percibidos. 
TABLA 1

Frecuencia absoluta y frecuencia relativa para los factores relacionados con el acercamiento a la disciplina

\begin{tabular}{|c|c|c|c|}
\hline Factores & Ítems & $\mathbf{F a}$ & Fr \\
\hline \multirow{8}{*}{ Razones } & Salud & 12 & $14 \%$ \\
\hline & Prescripción médica & 4 & $5 \%$ \\
\hline & Entrono Social & 5 & $6 \%$ \\
\hline & Gusto por el deporte & 33 & $38 \%$ \\
\hline & Aprovechamiento del tiempo libre & 8 & $9 \%$ \\
\hline & Autoestima & 10 & $11 \%$ \\
\hline & Mejora de la autoconfianza & 10 & $11 \%$ \\
\hline & Otra & 5 & $6 \%$ \\
\hline \multirow{7}{*}{ Acercamiento } & Radio & 0 & $0 \%$ \\
\hline & Televisión & 1 & $2 \%$ \\
\hline & Internet & 4 & $7 \%$ \\
\hline & Entorno social & 31 & $57 \%$ \\
\hline & Redes sociales & 3 & $6 \%$ \\
\hline & Entorno familiar & 1 & $2 \%$ \\
\hline & Otra & 14 & $26 \%$ \\
\hline \multirow{9}{*}{ Preferencia } & Bienestar & 11 & $8 \%$ \\
\hline & Socialización & 11 & $8 \%$ \\
\hline & Competencia & 27 & $21 \%$ \\
\hline & Reconocimiento & 14 & $11 \%$ \\
\hline & Búsqueda de metas & 22 & $17 \%$ \\
\hline & Mejora de la autoestima & 13 & $10 \%$ \\
\hline & Mejora de la autoconfianza & 10 & $8 \%$ \\
\hline & Ser ejemplo para todos & 22 & $17 \%$ \\
\hline & Otra & 1 & $1 \%$ \\
\hline \multirow{8}{*}{ Beneficios } & Salud & 25 & $19 \%$ \\
\hline & Bienestar & 14 & $11 \%$ \\
\hline & Conocer personas & 21 & $16 \%$ \\
\hline & Beneficios económicos & 12 & $9 \%$ \\
\hline & Autoestima & 16 & $12 \%$ \\
\hline & Mejora de la autoconfianza & 21 & $16 \%$ \\
\hline & Viajar & 18 & $14 \%$ \\
\hline & Otra & 4 & $3 \%$ \\
\hline
\end{tabular}


Como se logra evidenciar, el gusto por el deporte, el entorno social, aunado a la posibilidad de competir y el beneficio en salud que esta práctica deportiva trae consigo, son factores fundamentales que median en la elección del Para Powerlifting como disciplina en mujeres para atletas. Por otra parte, la Tabla 2 recoge los resultados de un primer conjunto de barreras y facilitadores percibidos por parte de las para atletas.

TABLA 2

Frecuencia absoluta y frecuencia relativa para los factores percibidos como barreras y facilitadores

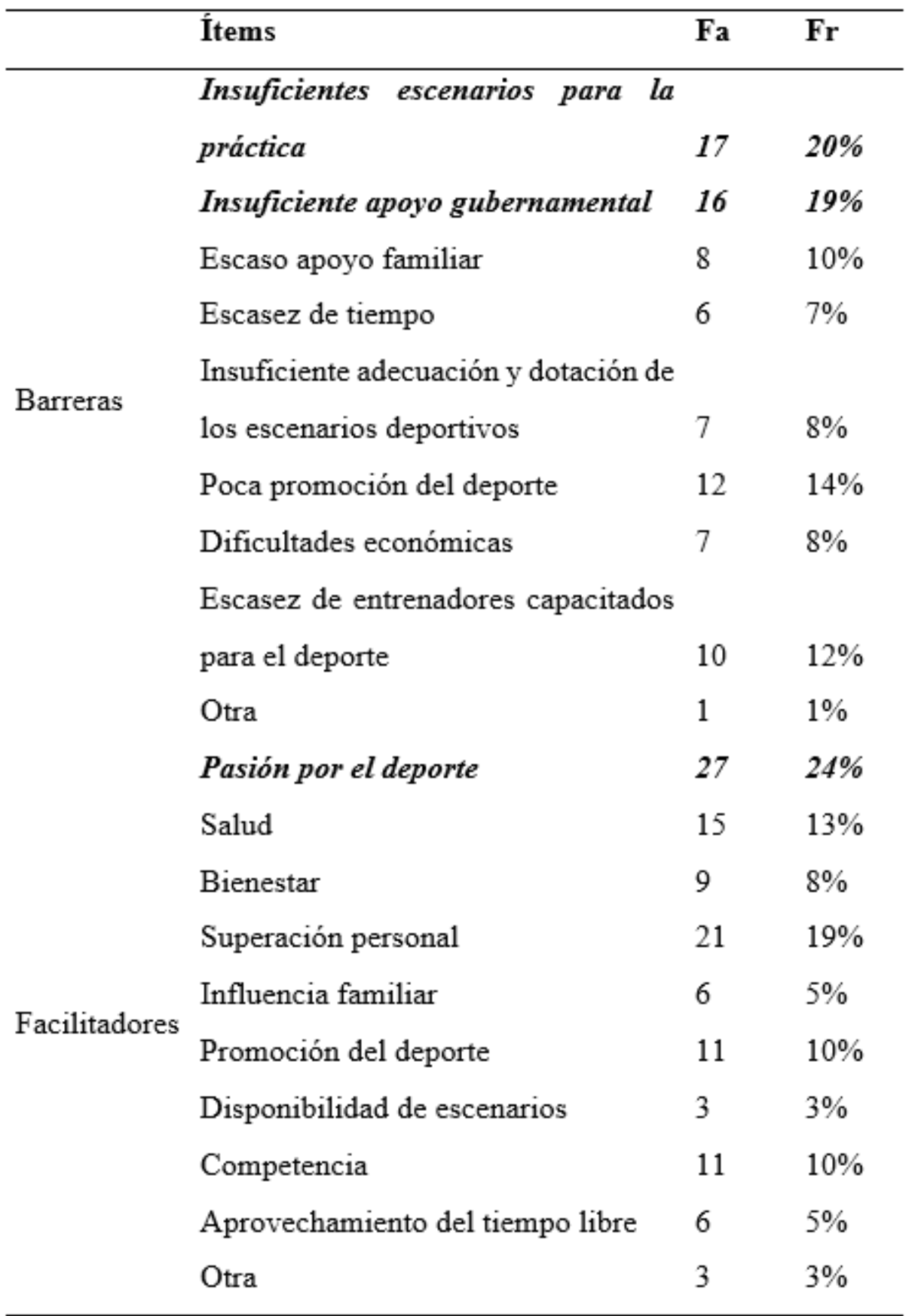

Fuente: elaboración propia

Es claro que las principales barreras percibidas en el desarrollo de la práctica del Para Powerlifting se encuentran la falta de escenarios para la práctica de la disciplina así como un insuficiente apoyo 
gubernamental; por su parte, se percibe la pasión por el deporte como una variable que facilita la práctica del mismo.

Finalmente, la Tabla 3 presenta las frecuencias relativas en la segunda parte del instrumento orientada a identificar cuáles de las barreras y/o facilitadores que tienen mayor importancia en la experiencia personal deportiva.

TABLA 3

Frecuencia relativa de los factores percibidos como barreras y facilitadores en la experiencia personal deportiva

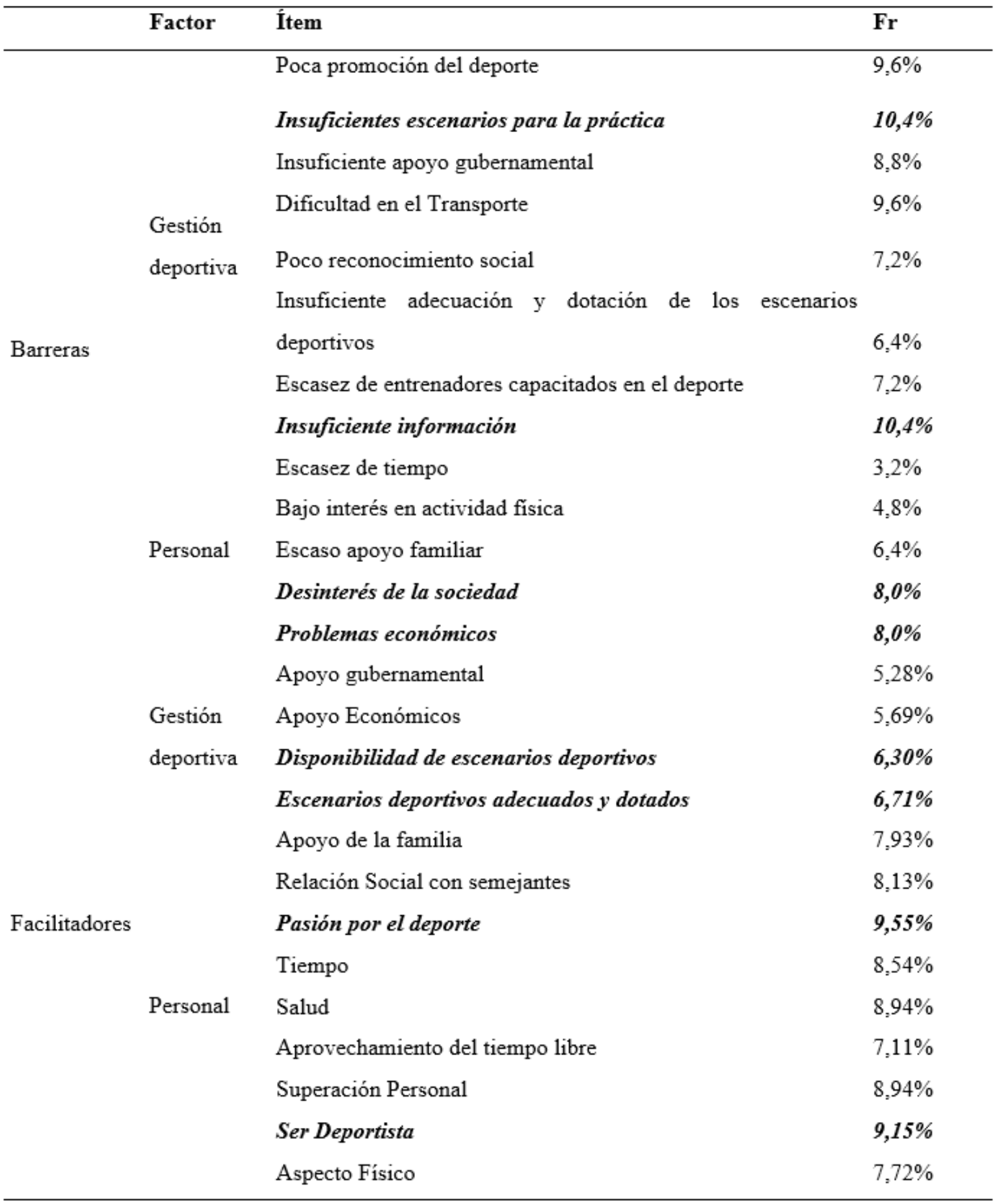


$\mathrm{Al}$ igual que en el caso de la Tabla 2, la principal barrera percibida en términos de gestión deportiva es la falta de escenarios para la práctica del deporte; adicionalmente, llama la atención la falta de información que existe alrededor del Para Powerlifting, lo cual puede repercutir en dificultades en la divulgación de esta disciplina al público en general. Esto va en línea con el desinterés de la sociedad como barrera en el factor personal, así como los problemas económicos para el normal desarrollo de actividades asociadas.

En términos de facilitadores, en línea con lo anterior los escenarios y equipos aparecen nuevamente como un aspecto clave en el desarrollo de la disciplina; por su parte, en términos personales, sobresale la pasión por el deporte, así como la vocación de deportista.

Se entienden como barreras aquellos limitantes que impiden la formación de una persona y como facilitadores aquellos que permiten a las personas desarrollarse en diferentes áreas. En este caso, estos se subdividieron en factores personales y de gestión deportiva. Las barreras más frecuentemente mencionadas se relacionan con la línea técnica, junto con la baja gestión deportiva al no tener escenarios adecuados para la práctica del deporte, pero esto no ha sido un impedimento para participar en deporte y llega a la obtención de altos logros. Se logra identificar que la práctica del Para Powerlifting es un motivador muy alto esto debido a su oportunidad de conseguir desarrollo profesional deportivo en esta disciplina. Las para atletas identifican los beneficios que trae la práctica cotidiana del deporte para la salud, tanto física como mental, adicionalmente conocen las consecuencias que puede traer a nivel salud la inactividad.

Factor de gestión. En la gestión deportiva se destaca la insuficiente adecuación y dotación de escenarios junto con los insuficientes escenarios para la práctica del Para Powerlifting; se toman como una barrera muy importante que pueden percibir las para atletas para realizar su práctica deportiva. Esta barrera coincide con los resultados presentados en el trabajo de Colomer, Alventosa y Granell, (2016) en el cual se observa que todo lo relacionado con gestión estructural y adecuaciones tienen un nivel muy alto de importancia. Igualmente, se percibe dificultad en el transporte y las adaptaciones adecuadas de este para las para atletas, tomándose como una barrera muy importante ya que la movilización en las ciudades para llegar a sus destinos pueden presentar inconvenientes. Esta particularidad se encuentra también en los resultados del trabajo de Abellán y Januário (2017), quienes destacaron que la movilidad en vehículos públicos es una dificultad significativa al no existir ninguno de estos medios con las adecuaciones óptimas para facilitar y dar comodidades a las personas con discapacidad en su tren inferior y que utilicen para movilizarse silla de ruedas. También se debe tener presente el recorrido que ellas tienen que hacer para llegar a esos puntos porque las calles por donde se movilizan las personas caminando o corriendo no tiene gran facilidad para ser usadas por personas con movilidad reducida, la infraestructura de una ciudad debe acoplarse y cumplir con las necesidades de todas las personas que se encuentran habitando para ofrecer calidad de vida. Teniendo en cuenta las respuestas recolectadas con el instrumento que se aplicó a las para atletas sobre su comodidad para movilizarse en la ciudad, se puede inferir que no hay accesibilidad suficiente en la infraestructura para las personas con discapacidad generando inconvenientes en la movilidad y el desplazamiento.

Otros factores de gestión deportiva que también son barreras con un nivel de importancia muy alto son la falta de apoyo gubernamental y la escasez de entrenadores que se encuentre capacitados en conocimientos sobre el entrenamiento y comprensión deportiva del Para Powerlifting. Esto es un impedimento para lograr los resultados personales y deportivos que las para atletas desean obtener; a su vez, estas barreras se relacionan con la económica, exhibiendo un apoyo escaso a nivel gubernamental que limita la adquisición de los recursos físicos y técnicos para mejorar y cumplir con las necesidades de esta población. En cuanto a los entrenadores bien formados, se requiere de un amplio conocimiento de la disciplina en aras de que las dosificaciones de la carga y las consideraciones específicas del deporte vayan en línea con las necesidades de rendimiento de las para atletas.

Factor personal. En el aspecto personal, se evidencia una marcada influencia por parte del aspecto económico, así como el desinterés de la sociedad por la práctica deportiva del Para Powerlifting; al respecto, Somerset y Hoare (2018) señalan que los padres, los maestros, los entrenadores y las personas encargadas de 
formular planes de acción, saben que una barrera clave para la práctica de los para deportes es el costo y el tiempo, y debería estar incluido en la política estatal para enfrentarlas; sin embargo, a nivel de los gobiernos no se toman las medidas adecuadas o de manera oportuna para lograr esta expansión deportiva dificultando la masificación de las disciplinas que las personas en con discapacidad pueden practicar competitivamente. En línea con lo anterior, Colomer et al., (2016), señalan que la falta de recursos económicos puede ser una barrera que impida la práctica de cualquier tipo de para deporte; según estos autores, esta barrera es superada por la mayoría de deportistas, aunque deja a muchas personas fuera de la actividad deportiva debido a que entorpece procesos como la movilización y obtención de elementos esenciales para la práctica deportiva. Por otra parte, la escasez de tiempo es una barrera muy importante para cumplir el horario del día a día, generando complicaciones o inconvenientes para la práctica deportiva (Abellán y Januário, 2017). La escasez de tiempo se toma como una barrera debido a que además de los periodos de los entrenamientos, también necesitan un lapso adicional para sus traslados hacia y desde sus sitios de entrenamiento para cumplir con los compromisos en el día a día de las para atletas.

No obstante, a pesar de todos estos inconvenientes, las para atletas han alcanzado beneficios gracias la práctica deportiva; esto concuerda con lo reportado por Yazicioglu, Yavuz, Salim y Tan (2012), quienes señalan que las personas con discapacidades físicas que participaban en deportes adaptados tenían puntuaciones de calidad y satisfacción con la vida significativamente más altas en comparación con las personas con discapacidades físicas que no participaban en ningún deporte; particularmente importante son las relaciones que pueden llegar a encontrar en el para deporte, las amistades dentro del deporte para personas con discapacidad pueden contribuir a la calidad de vida al promover estados afectivos positivos como sentimientos de alegría, satisfacción, inspiración, emoción y entusiasmo (Shapiro y Martin, 2010).

Se puede inferir que la mayoría de las para atletas llegaron al Para Powerlifting gracias a su entorno social (familia, amigos y allegados). También se ha identificado que la práctica del deporte les ha otorga un reconocimiento ante la sociedad. Por otra parte, se reconoce que la poca promoción del deporte es una barrera con mucha importancia, coincidiendo con Colomer et al., (2016), quienes indicaron que la información que reciben para esta asociación proviene de otras personas que conocen a otros con discapacidades similares, llegando entonces esta información principalmente de forma verbal.

\section{CONCLUSIONES Y RECOMENDACIONES}

Las barreras y facilitadores percibidas por las atletas del Para Powerlifting se encuentran ya sea en lo personal o en inconvenientes estructurales de las ciudades; teniendo presente lo anterior se recomienda realizar más estudios con relación a estos factores para lograr obtener más información debido a que no existen muchos estudios que presenten a fondo cuales son las barreras $\mathrm{y}$ facilitadores específicos en cada entorno.

Las redes sociales son un medio de comunicación masivo que no ha sido explotado poderosamente ya que, ninguna para atleta ha llegado a la práctica del Para Powerlifting por este medio. Se recomienda mejorar estos canales de información para conseguir más adherencia a disciplina deportiva y de este modo poder incrementar la participación de la mujer en este deporte. Se debe tener presente que algunas de ellas conocen el deporte por medio de las recomendaciones médicas sobre la práctica de la actividad física, pero no todas las mujeres del mundo cuentan con estas prescripciones, así que la mejora de comunicación a través de redes sociales es muy necesaria para mejorar la visibilidad de la disciplina que causaría un incremento de participantes mujeres, se recomienda que la promoción del deporte por medio de las redes sociales y publicidad externa como pancartas folletos entre otros sea constante.

La escasez de entrenadores capacitados en el deporte puede ser un limitante muy importante para la formación y desarrollo de las para atletas, es muy importante que los entrenadores que quieran formar parte de este tipo de deporte se encuentren con un nivel de competencia muy alto en relación con los métodos y 
estrategias de entrenamientos enfocados hacia las para atletas y de este modo pueda cumplir con los objetivos de rendimiento.

El escaso apoyo familiar puede ser otra barrera que limita la participación de las futuras Para atletas debido a que no les permitan realizar ningún tipo de actividad que genere grandes exigencias de fuerza física, como solución se pueden implementar talleres o campos de eventos que les permita compartir con mujeres para atletas y puedan adquirir un reflejo motivador.

El apoyo económico puede ser una limitante, pero puede ser solucionado con una buena publicidad y captar la atención de entidades que deseen apoyar este tipo de deportes.

La disponibilidad de escenarios deportivos y para la práctica, es una barrera y cuenta con un valor de impacto muy influyente para el desarrollo físico y para satisfacer las necesidades de las para atletas, por lo que se debe seguir influyendo en política pública a fin de garantizar la accesibilidad de las construcciones en general.

\section{REFERENCIAS}

Abellán, J., \& Januário, N. (2017). Barreras, facilitadores y motivos de la práctica deportiva de deportistas con discapacidad intelectual. Psychology, Society, \& Education., 9(3), 419-431. doi 10.25115/psye.v9i3.1023

Camargo, D. A., \& Forero, C. V. (2016) La inclusión deportiva en Colombia. Una mirada desde los actores. Estudio de caso. Revista de Investigación: Cuerpo, Cultura y Movimiento, 6(2), 143-165.

Colomer, J. U., Alventosa, P. M. \& Granell, J. C. (2016) Facilitadores y barreras para la actividad física en tiempo de ocio en alumnado universitario con discapacidad: un estudio cualitativo. Educación Física y Deporte, 35(1), 63-96. doi: / 10.17533/udea.efyd.v35n1a03

Diaz, R., Miller, E. K., Kraus, E., \& Fredericson, M. (2019). Impact of Adaptive Sports Participation on Quality of Life. Sports medicine and arthroscopy review, 27(2), 73-82. https://doi.org/10.1097/JSA.0000000000000242

Evans, M. B., Shirazipour, C. H., Allan, V., Zanhour, M., Sweet, S. N., Martin Ginis, K. A., \& Latimer-Cheung, A. E. (2018). Integrating insights from the parasport community to understand optimal Experiences: The Quality Parasport Participation Framework. Psychology of Sport and Exercise, 37, 79-90. doi: /10.1016/ j.psychsport.2018.04.009

Farzaneh, S., Rahavi, R., Khalili, S. S., Khalili, P. \& Ranawat, V. (2021) Identifying Barriers to Women's Participation in Sports Activities in both Urban and Rural Communities. International Journal of Human Movement and Sports Sciences, 9(3), 536 - 542. doi: 10.13189/saj.2021.090320.

Hernández, R., Fernández, C. \& Baptista, M. P. (2014) Metodología de la Investigación. México D.C.: McGraw-Hill

Jaarsma, E. A., Dijkstra, P. U., Geertzen, J. H. \& Dekker, R. (2014) Barriers and facilitators of sports participation for people with physical disabilities: a systematic review. Scandinavian Journal of Medicine \& Science in Sports, 24(6), p. 11. doi:10.1111/sms.12218

Jefferies, P., Gallagher, P. \& Dunne, S. (2012). The Paralympic athlete: a systematic review of the psychosocial literature. Prosthetics and Orthotics International, 36(3), 278-289. https://doi.org/10.1177/03093646124501 84

McLoughlin, G., Weisman, C., Castaneda, Y., Gwin, C. \& Graber, K. (2017). Sport Participation for Elite Athletes With Physical Disabilities: Motivations, Barriers, and Facilitators. Adapted Physical Activity Quarterly, 34(4), 421-441. doi: 10.1123/apaq.2016-0127

Moya, R. M. (2014) Deporte adaptado. Tomado de: https://www.imserso.es/InterPresent2/groups/imserso/docum ents/binario/deporteadaptado.pdf

Organización de Naciones Unidas (2007) Women, gender equality and sport. Disponible en: https://www.un.org/w omenwatch/daw/public/Women\%20and\%20Sport.pdf

Ruiz, S. (2012) Deporte paralímpico: una mirada hacia el futuro. Revista U.D.C.A Actualidad \& Divulgación Cientifica, 15(sup), 97-104. 
Shapiro, D. R. \& Martin, J. (2010). Athletic identity, affect, and peer relations in youth athletes with physical disabilities. Disability and Health Journal, 3(2), 79-85. doi: 10.1016/j.dhjo.2009.08.004

Somerset, S. \& Hoare, D. J. (2018) Barriers to voluntary participation in sport for children: a systematic review. BMC pediatrics, $18(47)$. doi:10.1186/s12887-018-1014-1

Wilde, K. (2007). Women in Sport : Gender Stereotypes in the Past and Present. Disponible en: http://wgst.athaba scau.ca/awards/broberts/forms/Wilde.pdf

World Para Powerlifting (s.f.) History of Para Powerlifting. Disponible en: https://www.paralympic.org/powerliftin g/about

Yagüe, L.C. (2008) El deporte olímpico de las personas con discapacidad. Citius, Altius, Fortius: Humanismo, Sociedad y Deporte: Investigaciones y Ensayos, 133-145.

Yazicioglu, K., Yavuz, F., Salim, A. \& Tan, A. K. (2012) Influence of adapted sports on quality of life and life satisfaction in sport participants and non-sport participants with physical disabilities. Disability and Health Journal, 5(4), 249-253. doi: 10.1016/j.dhjo.2012.05.003 